\title{
Combination of BIBW2992 and ARQ 197 is effective against erlotinib-resistant human lung cancer cells with the EGFR T790M mutation
}

\author{
GEPING QU, CHANGTING LIU, BAOJUN SUN, CHANGXI ZHOU, ZHIJIAN ZHANG and PENG WANG \\ Nanlou Respiratory Diseases Department, The Chinese People's Liberation Army (PLA) \\ General Hospital, Beijing 100853, P.R. China
}

Received February 21, 2014; Accepted April 24, 2014

DOI: $10.3892 / o r .2014 .3178$

\begin{abstract}
Although the EGFR tyrosine kinase inhibitors (EGFR-TKI) erlotinib and gefitinib have shown marked effects against EGFR-mutated lung cancer, patients acquire resistance by various mechanisms, including the EGFR T790M mutation and Met induction, consequently suffering relapse. Thus, novel agents to overcome EGFR-TKI resistance are urgently needed. We aimed to investigate the inhibitory effects of a combination of BIBW2992 (irreversible EGFR inhibitor)/ARQ 197 (MET inhibitor) on the human lung adenocarcinoma cell line H1975. H1975 cells (harboring a T790M mutation in EGFR) were treated with erlotinib, BIBW2992 or ARQ 197 separately or with combinations of erlotinib/ARQ 197 or BIBW2992/ ARQ 197. Cell growth, apoptosis and cell cycle distribution were evaluated by MTT assay, Annexin V/propidium iodide (PI) double staining and flow cytometry, respectively. EGFR, MET, AKT, ERK and the respective phosphorylated counterparts were detected by western blot analysis. Pathwayspecific knockdown of MET and/or EGFR kinase signaling was achieved by siRNA interference. H1975 cells displayed EGFR and MET activation, and were resistant to erlotinib. The BIBW2992/ARQ 197 combination significantly inhibited growth, induced cell cycle arrest and apoptosis, and altered the phosphorylation of EGFR, MET, AKT and ERK1/2 in the H1975 cells. Phosphorylation of AKT and ERK1/2, downstream effectors of the EGFR and MET pathways, was not affected by the other tested treatments. Finally, knockdown of MET and/or EGFR in the H1975 cells confirmed the enhanced downstream inhibition of both MET and EGFR pathways. Combination of an irreversible EGFR inhibitor and MET inhibitor is effective in controlling H1975 cells with acquired
\end{abstract}

Correspondence to: Dr Changting Liu, Nanlou Respiratory Diseases Department, The Chinese People's Liberation Army (PLA) General Hospital, Beijing 100853, P.R. China

E-mail: liuchangtingmed@163.com

Key words: lung cancer, erlotinib resistance, irreversible EGFR inhibitor, MET inhibitor, combination therapy resistance to erlotinib, by a mechanism involving the downregulation of $\mathrm{PI} 3 \mathrm{~K} / \mathrm{AKT}$ and MEK/ERK signaling pathways.

\section{Introduction}

Epidermal growth factor receptor (EGFR) tyrosine kinase inhibitors (TKI) have shown marked therapeutic effects against non-small cell lung cancer (NSCLC) with EGFR activating mutations, such as exon 19 deletions and L858R point mutations (1). However, acquired resistance to EGFRTKIs develops in almost all patients, usually within 1 year, thus, limiting improvement in patient outcomes $(2,3)$. Approximately $50 \%$ of patients resistant to the first generation EGFR-TKIs gefitinib or erlotinib (also known as reversible EGFR-TKIs) present tumors with a secondary mutation in exon 20 of EGFR, which involves the substitution of threonine at position 790 by methionine (T790M) in the tyrosine kinase functional domain; $20 \%$ have tumors with bypass signaling caused by proto-oncogene Met amplification or overexpression which activates downstream pathways, including phosphatidylinositol 3-kinase/protein kinase B (PI3K/AKT) and mitogen-activated protein kinase kinase/extracellular signal-regulated kinase (MEK/ERK) $(4,5)$.

The T790M mutation was shown to confer resistance by increasing EGFR affinity for ATP relative to that for first generation EGFR-TKIs, resulting in continuous activation of downstream pro-survival signaling pathways, such as PI3K/AKT and MEK/ERK (6). The second generation EGFR-TKIs (also known as irreversible EGFR-TKIs), such as BIBW2992, were designed to covalently bind the kinase domain and are, therefore, less affected by the increase in ATP-binding affinity compared with the reversible inhibitors (7). However, $\mathrm{IC}_{50}$ values of irreversible EGFR-TKIs are $>400$ times higher in NSCLC cell lines with the T790M mutation than in NSCLC cells without the T790M mutation, markedly diminishing the clinical value of irreversible EGFR-TKIs (8). Several studies have demonstrated that although MET-TKI shows marginal efficacy in NSCLC cell lines with the T790M mutation when administered alone, its combinations with gefitinib/erlotinib are effective in mutated cells (9-11). These observations might be explained by the fact that many growth factor signaling pathways overlap and interact with each other, suggesting 
a redundancy in cell signaling. For instance, activation of one tyrosine kinase receptor may co-activate the downstream signaling pathway of other tyrosine kinase receptors. Therefore, strategies to interrupt receptor cross-signaling or to target more than one pathway may result in increased effects on tumor inhibition.

To the best of our knowledge, no previous study has reported the effects of a combination of BIBW2992 and ARQ 197 (MET inhibitor) on proliferation, apoptosis and downstream signaling pathways of EGFR/MET in NSCLC cell lines. Therefore, we investigated the effects of such a combination (BIBW2992 and ARQ 197) on the NSCLC cell line H1975 harboring an EGFR T790M mutation, aimed at the two molecular mechanisms of acquired resistance to reversible EGFR-TKIs as mentioned above. Our results showed that the BIBW2992 and ARQ 197 combination inhibited cell growth, induced cell apoptosis and cell cycle arrest at the $G_{0} / G_{1}$ phase, and reduced the phosphorylation of AKT and ERK1/2, primary downstream effectors of the EGFR and c-MET signaling pathways.

\section{Materials and methods}

Cell culture, chemicals and antibodies. Human lung adenocarcinoma cell line H1975 was purchased from the Cell Biology Institute of the Chinese Academy of Sciences, Beijing, China. Cells were cultured in RPMI-1640 (Invitrogen, Carlsbad, CA, USA) supplemented with $10 \%$ FBS, penicillin (100 units/ml) and streptomycin $(100 \mathrm{mg} / \mathrm{ml})$ at $37^{\circ} \mathrm{C}$ in a humidified environment containing $5 \% \mathrm{CO}_{2}$.

BIBW2992 and ARQ 197 were purchased from Sigma Chemical (St. Louis, MO, USA). Erlotinib hydrochloride was obtained from Roche China. These drugs were dissolved in DMSO and used at the indicated concentrations. The Annexin V-PI double staining apoptosis detection kit was purchased from Jingmei Biotech Co., Ltd. (Shanghai, China).

Genomic studies of the EGFR and MET genes. Genomic DNA was purified from H1975 cells using the Qiagen DNAeasy Kit (Qiagen, Shanghai, China) according to the manufacturer's instructions. Mutation analysis of the EGFR, MET and KRAS genes was carried out by direct sequencing after OneStep reverse transcriptase-PCR (RT-PCR) using the Qiagen OneStep Reverse Transcription-PCR kit (Qiagen).

MET gene copy number detection. Genomic copy number variation of the MET gene in H1975 cells was assessed using real-time PCR with Power SYBR-Green PCR Master Mix (Applied Biosystems, Shanghai, China) on an ABI PRISM 7900-HT System. PCR reactions were set following the standard $\Delta \mathrm{C}_{\mathrm{T}}$ method according to the manual. MET q-PCR primers were purchased from ABI (ABI assay no. Hs01565582_g1). RNaseP was used as a reference gene.

Cytotoxicity assays. Cell viability was assessed using the MTT (3-(4,5-dimethylthiazol-2-yl)-2,5-diphenyltetrazolium bromide) colorimetric assay (12). Briefly, cells were seeded in 96-well plates at a density of $1 \times 10^{4}$ cells/well for $16-20 \mathrm{~h}$. After treatment for $48 \mathrm{~h}$ with erlotinib $(2 \mu \mathrm{M})$, BIBW2992 $(1 \mu \mathrm{M})$, ARQ $197(2 \mu \mathrm{M})$, and the $2 \mu \mathrm{M}$ erlotinib/2 $\mu \mathrm{M}$ ARQ 197 and $1 \mu$ M BIBW2992/2 $\mu$ M ARQ 197 combinations, the medium was removed and the cells were incubated with $0.5 \mathrm{mg} / \mathrm{ml}$ MTT in complete medium for $4 \mathrm{~h}$. The absorbance was measured at $565 \mathrm{~nm}$ using an OptiMax plate reader (GE Healthcare, Shanghai, China), and the viability was expressed as a percentage relative to the control cells.

Apoptosis assay. Apoptosis rates were determined by staining cells using an Annexin V-fluorescein isothiocyanate (FITC) and propidium iodide (PI) kit (Jingmei Biotechnology Co., Ltd.) according to the manufacturer's instructions. Briefly, $1 \times 10^{6} \mathrm{H} 1975$ cells were incubated in the presence of erlotinib $(2 \mu \mathrm{M})$, BIBW2992 $(1 \mu \mathrm{M})$, ARQ $197(2 \mu \mathrm{M})$, and the $2 \mu \mathrm{M}$ erlotinib/2 $\mu \mathrm{M}$ ARQ 197 and $1 \mu \mathrm{M}$ BIBW2992/2 $\mu \mathrm{M}$ ARQ 197 combinations. After $48 \mathrm{~h}$ of incubation, cells were washed twice with phosphate-buffered solution (PBS; pH 7.4), resuspended in $500 \mu \mathrm{l}$ binding buffer before addition of $5 \mu \mathrm{l}$ Annexin $\mathrm{V}$ and $1 \mathrm{mg} / \mathrm{ml}$ PI, and analyzed on an LSR flow cytometer (BD Biosciences, San Jose, CA, USA) using CellQuest software (BD Biosciences). Early apoptotic cells were positive for Annexin V and negative for PI while late apoptotic cells were positive for both Annexin V and PI.

Analysis of cell cycle distribution. $\mathrm{H} 1975$ cells $\left(1 \times 10^{6}\right)$ were seeded in p60 Petri dishes in complete medium and incubated for 16-20 h. After treatment with erlotinib $(2 \mu \mathrm{M})$, BIBW2992 $(1 \mu \mathrm{M})$, ARQ $197(2 \mu \mathrm{M})$, and $2 \mu \mathrm{M}$ erlotinib/2 $\mu \mathrm{M}$ ARQ 197 and $1 \mu \mathrm{M}$ BIBW2992/2 $\mu \mathrm{M}$ ARQ 197 combinations for $48 \mathrm{~h}$, cells were collected and fixed with ice-cold $70 \%$ ethanol overnight at $-20^{\circ} \mathrm{C}$. Upon centrifugation, cell pellets were treated with $4 \mathrm{mg} / \mathrm{ml}$ PI solution containing $1 \%$ Triton X-100 and $100 \mathrm{mg} / \mathrm{ml}$ RNase for $30 \mathrm{~min}$. To avoid cell aggregation, cell suspensions were filtered with nylon membranes (BD Biosciences), and the samples were analyzed on an LSR flow cytometer using CellQuest software. A minimum of $1 \times 10^{4}$ cells were analyzed for DNA content, and the percentages of cells in various cell cycle phases were quantified using the ModFit LT ver. 3.0 (BD Biosciences).

Western blot analysis. H1975 cells were incubated in the presence of erlotinib $(2 \mu \mathrm{M})$, BIBW2992 $(1 \mu \mathrm{M})$, ARQ 197 $(2 \mu \mathrm{M})$, and $2 \mu \mathrm{M}$ erlotinib/2 $\mu \mathrm{M}$ ARQ 197 and $1 \mu \mathrm{M}$ BIBW2992/2 $\mu \mathrm{M}$ ARQ 197 combinations for $48 \mathrm{~h}$, collected and lysed in ice-cold cell extraction buffer (Life Technologies, Shanghai, China). Protein concentrations were determined using the BCA protein assay kit (Pierce, Rockford, IL, USA), and equal protein amounts (50-100 $\mu \mathrm{g} /$ well) were subjected to electrophoresis on $12 \%$ SDS-polyacrylamide gels. After electrophoretic transfer of proteins onto nitrocellulose membranes, samples were sequentially incubated with primary antibodies and goat anti-rabbit secondary antibodies conjugated to horseradish peroxidase (Cell Signaling Technology Inc., Beverly, MA, USA). Primary antibodies raised in rabbit against human EGFR, p-EGFR (Y1068), MET and p-MET (Y1234/1235) were purchased from Santa Cruz Biotechnology (Santa Cruz, CA, USA); rabbit anti-human AKT, p-AKT (S473), ERK1/2, p-ERK1/2 (T202/Y204) and $\beta$-actin antibodies were purchased from Abcam (Cambridgeshire, UK).

Finally, protein bands were detected using the enhanced chemiluminescence kit (ECL; Pierce), and the membranes were exposed to X-ray film and imaged. 


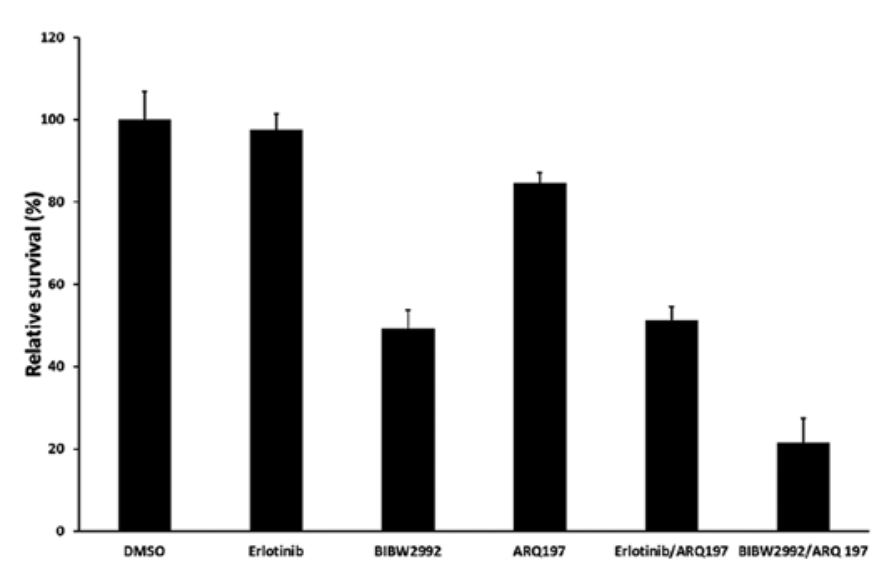

Figure 1. Combination of BIBW2992 and ARQ 197 results in pronounced H1975 cell growth inhibition. H1975 cells were treated with $2 \mu \mathrm{M}$ erlotinib, $1 \mu \mathrm{M}$ BIBW2992, $2 \mu \mathrm{M}$ ARQ 197, and the $2 \mu \mathrm{M}$ erlotinib/2 $\mu \mathrm{M}$ ARQ 197 and $1 \mu \mathrm{M}$ BIBW2992/2 $\mu \mathrm{M}$ ARQ 197 combinations for $48 \mathrm{~h}$, and cell viability was evaluated by MTT assay. Data are the percentages of viable cells compared with the untreated controls (DMSO) and are represented as means \pm SD. Results were obtained from three independent experiments.

RNA interference. Duplexed Stealth RNA interference (Invitrogen) against MET and Stealth RNA Interference Negative Control Low GC Duplex 3 (Invitrogen) were used for RNA interference assays. Briefly, $1 \times 10^{5} \mathrm{H} 1975$ cells suspended in $2 \mathrm{ml}$ antibiotic-free medium were seeded in 6-well plates and incubated at $37^{\circ} \mathrm{C}$ for $24 \mathrm{~h}$. Then, cells were transfected with small interfering RNA (siRNA; 250 pmol) or scramble RNA (siSCR) using Lipofectamine 2000 (5 $\mu$ l) (Invitrogen) following the manufacturer's instructions. After $48 \mathrm{~h}$ of incubation, cells were used in proliferation and apoptosis assays as described above. MET and EGFR knockdown were confirmed by western blot analysis, and the siRNA sequences were as follows: MET forward, 5'-UCCAGAAGAUCAGUUUCCUA AUUCA-3' and reverse, 5'-UGAAUUAGGAAACUGAUCU UCUGGA-5'; EGFR forward, 5'-UUUAAAUUCACCAAUA CCUAUUCCG-3' and reverse, 5'-CGGAAUAGGUAUUGG UGAAUUUAAA-5'.

Statistical analysis. Data are expressed as means \pm SD and were nalyzed by the Student's t-test or one way analysis of variance (ANOVA) for comparison between multiple groups. $\mathrm{P}<0.05$ was considered to indicate a statistically significant result.

\section{Results}

EGFR and MET genotypes in the H1975 cells. Direct DNA sequencing showed L858R and T790M mutations in the EGFR gene of the H1975 cells, whereas no mutations were detected in the MET and KRAS genes in this cell line. Using qPCR analysis, we found that MET had 1.1 copies in H1975 cells.

Effects of the BIBW2992/ARQ 197 combination on H1975 cell growth. H1975 cells were incubated with erlotinib, BIBW2992, ARQ 197 and the combinations of erlotinib/ARQ 197 and BIBW2992/ARQ 197 for $48 \mathrm{~h}$, respectively, and cell proliferation was evaluated by MTT assays. Erlotinib and ARQ 197 used individually exhibited little cytotoxicity on H1975
Table I. Effects of the BIBW2992/ARQ 197 combination on the cell cycle distribution of $\mathrm{H} 1975$ cells.

\begin{tabular}{lccc}
\hline & \multicolumn{3}{c}{ Cell cycle phase (\%) } \\
\cline { 2 - 4 } Drugs & $\mathrm{G}_{0} / \mathrm{G}_{1}$ & $\mathrm{~S}$ & $\mathrm{G}_{2} / \mathrm{M}$ \\
\hline DMSO & $38.6 \pm 3.1$ & $42.1 \pm 2.6$ & $19.3 \pm 3.3$ \\
Erlotinib & $38.9 \pm 2.8$ & $43.8 \pm 3.7$ & $17.3 \pm 1.8$ \\
BIBW2992 & $40.5 \pm 3.4$ & $33.8 \pm 2.2$ & $25.7 \pm 4.6$ \\
ARQ 197 & $43.1 \pm 0.8$ & $32.2 \pm 1.6$ & $24.7 \pm 3.5$ \\
Erlotinib/ARQ 197 & $46.4 \pm 2.3$ & $30.8 \pm 4.4$ & $22.8 \pm 1.7$ \\
BIBW2992/ARQ 197 & $72.9 \pm 3.7^{\text {a }}$ & $15.8 \pm 3.1$ & $11.3 \pm 0.6$ \\
\hline
\end{tabular}

H1975 cells were treated with $2 \mu \mathrm{M}$ erlotinib, $1 \mu \mathrm{M}$ BIBW2992, $2 \mu \mathrm{M}$ ARQ 197, and the $2 \mu \mathrm{M}$ erlotinib/2 $\mu \mathrm{M}$ ARQ 197 and $1 \mu \mathrm{M}$ BIBW2992/2 $\mu$ M ARQ 197 combinations for $48 \mathrm{~h}$. Control cells were treated with DMSO. After treatment, cells were stained with PI and analyzed by flow cytometry. Each value represents mean \pm SD obtained from three independent experiments. ${ }^{\mathrm{a}} \mathrm{P}<0.01$, compared with the other experimental groups.

cells, with 97.5 and $84.6 \%$ viable cells, respectively, while BIBW2992 alone and the erlotinib and ARQ 197 combination showed moderate cytotoxicity (with 49.2 and $57.3 \%$ viable cells, respectively; $\mathrm{P}<0.05$ compared with DMSO). Notably, treatment with the BIBW2992/ARQ 197 combination resulted in pronounced growth inhibition of H1975 cells, with only $21.5 \%$ viable cells detected $(\mathrm{P}<0.01$ compared with DMSO and the erlotinib/ARQ 197 combination) (Fig. 1).

Effects of the BIBW2992/ARQ 197 combination on apoptosis in the $H 1975$ cells. H1975 cells were treated as described for the cell viability assays, and apoptosis was assessed by Annexin V-PI double staining. Annexin V can be used to detect externalization of phosphatidylserine during the progression of apoptosis, therefore identifying cells in early apoptosis. H1975 cells treated with erlotinib displayed an early apoptosis rate of $3.0 \%$, similar to the controls, indicating $\mathrm{H} 1975$ cell resistance to erlotinib. Treatment with BIBW2992, ARQ 197 and the erlotinib/ARQ 197 combination resulted in 31.2, 24.1 and $26.4 \%$ early apoptosis, respectively $(\mathrm{P}<0.001$ compared with the control cells). Importantly, the early apoptosis rate of $\mathrm{H} 1975$ cells after treatment with the combination of BIBW2992/ ARQ 197 was highest at $68.4 \%$ as shown in Fig. 2 ( $\mathrm{P}<0.01$ compared with the control, erlotinib, BIBW2992, ARQ 197 and erlotinib/ARQ 197 combination). These data indicate that the BIBW2992/ARQ 197 combination was much stronger in inducing apoptosis in H1975 cells in comparison with BIBW2992, ARQ 197 and the erlotinib/ARQ 197 combination.

Effects of BIBW2992/ARQ 197 combination on the $H 1975$ cell cycle. $\mathrm{H} 1975$ cells were treated as described above, and the percentages of cells in the cell cycle phases were assessed by flow cytometry. After treatment with erlotinib, BIBW2992, ARQ 197 and the combination of erlotinib/ARQ 197, slightly more $\mathrm{H} 1975$ cells were detected in the $\mathrm{G}_{0} / \mathrm{G}_{1}$ phase in comparison with the control samples, but with no statistical significance. However, $72.9 \%$ of the H1975 cells were found in 


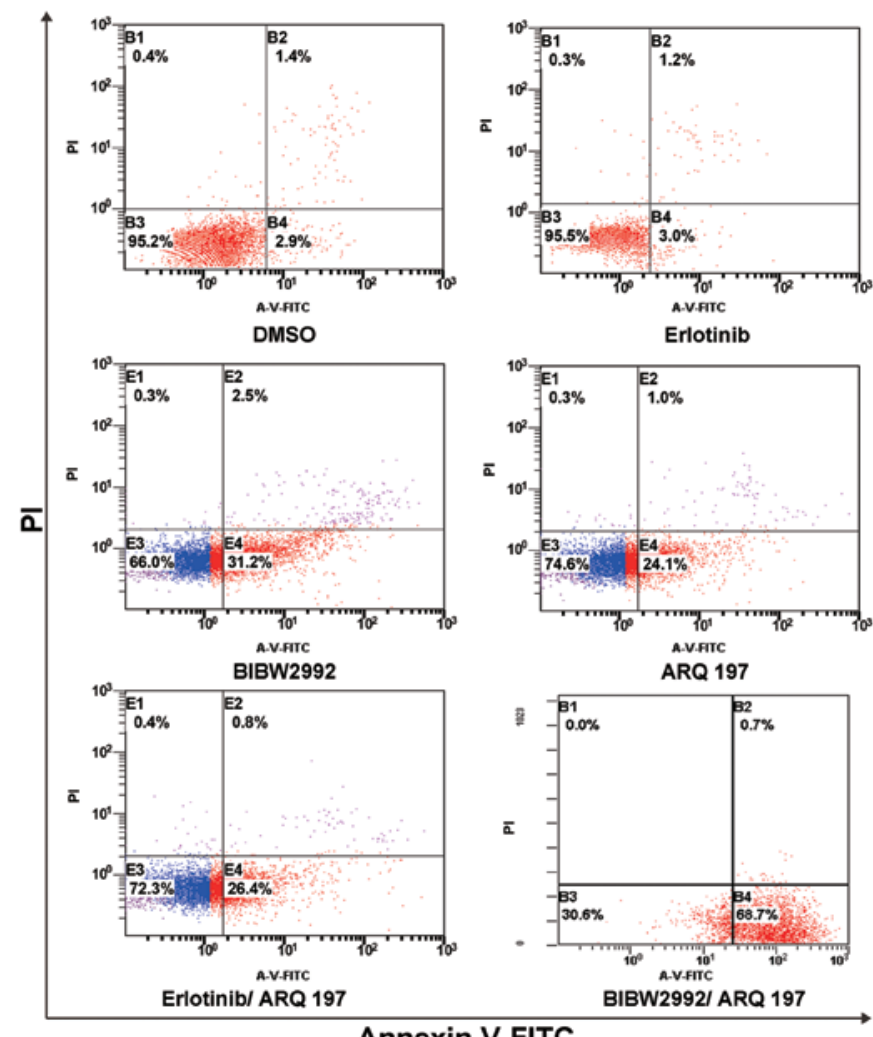

Annexin V-FITC

Figure 2. Combination of BIBW2992 and ARQ 197 display the most pronounced effects on H1975 cell apoptosis. H1975 cells were treated with $2 \mu \mathrm{M}$ erlotinib, $1 \mu \mathrm{M}$ BIBW2992, $2 \mu \mathrm{M}$ ARQ 197 and the $2 \mu \mathrm{M}$ erlotinib/2 $\mu \mathrm{M}$ ARQ 197 and $1 \mu \mathrm{M}$ BIBW2992/2 $\mu \mathrm{M}$ ARQ 197 combinations for $48 \mathrm{~h}$. Control cells were treated with DMSO. Cells were simultaneously stained with Annexin V-fluorescein isothiocyanate (FITC) and propidium iodide (PI), and the apoptosis rates were assessed by flow cytometry. Cells positive for Annexin V-FITC and negative for PI were in early apoptosis. Results were obtained from three independent experiments.

the $\mathrm{G}_{0} / \mathrm{G}_{1}$ phase after treatment with the BIBW2992/ARQ 197 combination; a rate much higher than that in any other treatment group (Table I; P<0.01 compared with the controls, erlotinib, BIBW2992, ARQ 197 and the erlotinib/ARQ 197 combination).

Effects of the BIBW2992/ARQ 197 combination on the expression of EGFR, MET and downstream effectors. $\mathrm{H} 1975$ cells were treated as described above, and the proteins were detected by western blot analysis. As shown in Fig. 3, control cells robustly expressed total MET and phosphorylated MET. Combined with our gene analysis results, these data confirmed MET activation with no genomic amplification or mutation in H1975 cells. In addition, erlotinib did not significantly affect EGFR, MET, AKT or ERK1/2. Our data showed decreased levels of phosphorylated EGFR after treatment with BIBW2992 and reduced levels of phosphorylated MET in the presence of the erlotinib/ARQ 197 combination and ARQ 197 alone. Of note, expression levels of total and phosphorylated AKT or ERK1/2 were not markedly altered in these groups. In contrast to the control and other treatments, expression levels of phosphorylated EGFR, MET, AKT and ERK1/2 were significantly decreased after treatment with the BIBW2992/ ARQ 197 combination (Fig. 3).

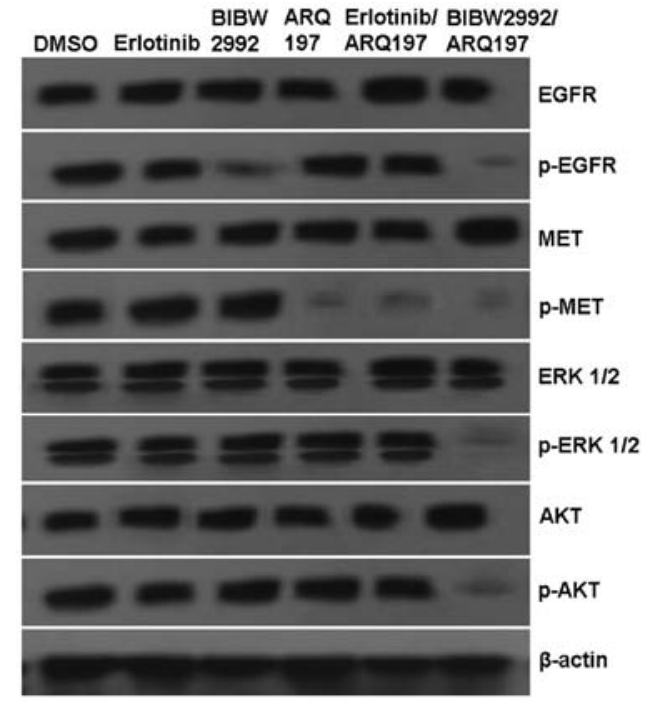

Figure 3. Combination of BIBW2992 and ARQ 197 induces simultaneous inhibition of EGFR and c-MET downstream effectors in H1975 cells. H1975 cells were treated with $2 \mu \mathrm{M}$ erlotinib, $1 \mu \mathrm{M}$ BIBW2992, $2 \mu \mathrm{M}$ ARQ 197, and the combinations of $2 \mu \mathrm{M}$ erlotinib/2 $\mu \mathrm{M}$ ARQ 197 and $1 \mu \mathrm{M}$ BIBW2992/2 $\mu \mathrm{M}$ ARQ 197 for $48 \mathrm{~h}$. Control cells were treated with DMSO. Whole cell lysates were collected for western blot analyses. $\beta$-actin protein levels were evaluated as loading controls.

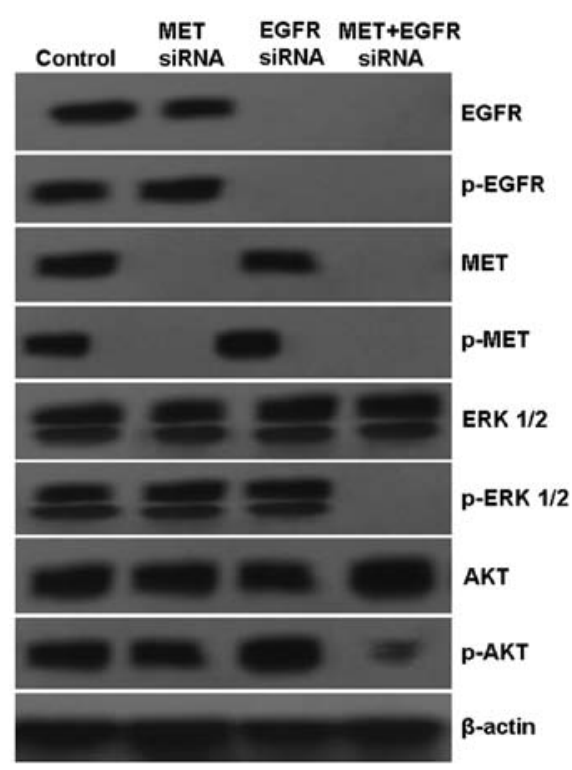

Figure 4. Simultaneous knockdown of MET and EGFR signaling in H1975 cells results in the enhanced inhibition of downstream signal molecules. H1975 cells were treated with siRNAs specifically targeted against mRNAs of MET, EGFR or both MET and EGFR. Cells were analyzed by western bloting using antibodies against molecules of the MET and EGFR signaling pathways, including the downstream pro-survival effectors AKT and ERK. $\beta$-actin protein levels were evaluated as loading controls.

Effects of specific EGFR and MET downregulation on growth and apoptosis in H1975 cells. To further validate this combination dual TKI strategy, H1975 cells were subjected to pathway-specific siRNA knockdown of MET and EGFR kinase signaling pathways, either alone or in combination. After $48 \mathrm{~h}$ of culture, optimal inhibition of downstream signaling molecules (phosphorylated-AKT and ERK1/2) was achieved, 


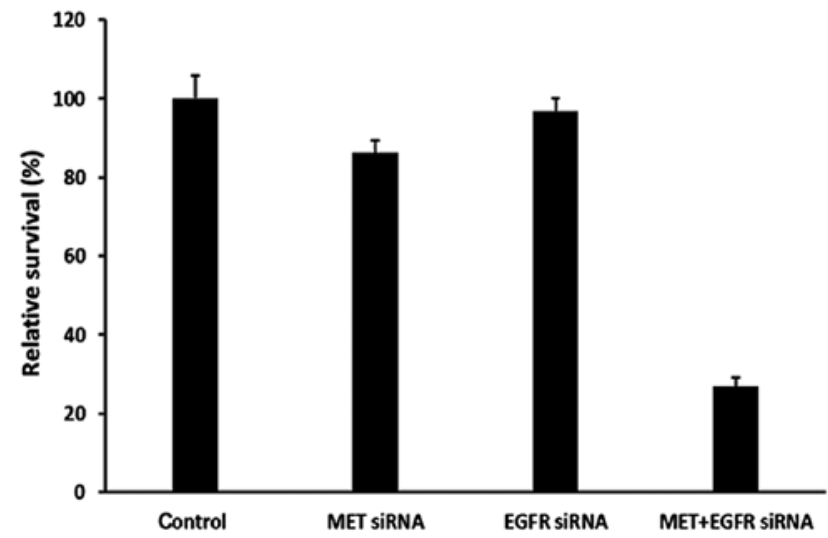

Figure 5. Simultaneous knockdown of MET and EGFR signaling results in pronounced cell growth inhibition in H1975 cells. H1975 cells were treated with siRNAs specifically targeted against mRNAs of MET, EGFR or both MET and EGFR. Control cells were transfected with empty vectors. Cell survival was measured by MTT assay. Data are percentages of viable cells compared with the controls and are represented as means \pm SD. Results were obtained from three independent experiments.

as observed with siRNA knockdown of both MET and EGFR targets, compared with single target knockdown (Fig. 4). We found a much higher cell growth inhibition and increased early cell apoptosis rates after knockdown of both MET and EGFR targets compared with single target knockdown (Figs. 5 and 6). Taken together, these data indicate that cooperative enhanced inhibition using dual TKIs against the MET and EGFR pathways may constitute an effective treatment strategy to inhibit H1975 cells with acquired T790M-EGFR-mediated TKI resistance.

\section{Discussion}

In the early days of anticancer therapy, the observation that tumors are hyperproliferative lesions prompted the development of antimitotic and cytostatic compounds with the hope that they would function indiscriminately on each cancer type. As it became evident that cancers have multiple etiologies, and that neoplastic progression is associated with a combination of genetic and epigenetic alterations, the task of developing therapies suitable for treatment of the full spectrum of cancers appeared almost impossible (13).

To date, it is well established that inactivation of individual oncogenes can block tumor growth and even lead to tumor regression; despite the multitude of genetic alterations harbored by transformed cells (14). These findings have given an unprecedented clinical value to the concept that considers cancer a disease of genes, allowing a novel classification of tumors based on the presence of defined genetic lesions. Classical histopathological diagnosis is still important to evaluate the extent of phenotypic aggressiveness, but personalized molecular diagnosis is needed to understand whether a tumor in a given patient carries a particular genetic alteration that could be targeted by therapy (15).

Recent prospective studies have demonstrated that the EGFR-TKIs gefitinib and erlotinib are associated with a high response rate and prolong progression-free survival in patients with EGFR activating mutant lung cancer. Responders to these

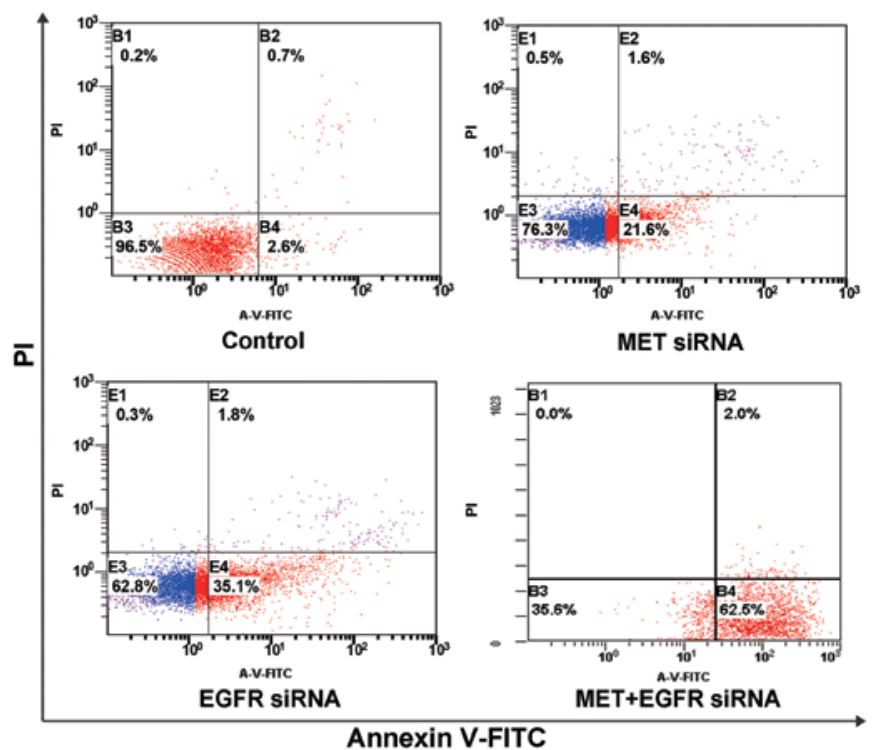

Figure 6. Simultaneous knockdown of MET and EGFR signaling results in pronounced apoptotic effects in H1975 cells. H1975 cells were treated with siRNAs specifically targeted against mRNAs of MET, EGFR or both MET and EGFR. Control cells were transfected with empty vectors. Cells were stained with Annexin V-fluorescein isothiocyanate (FITC) and propidium iodide (PI), and the apoptosis rates were assessed by flow cytometry. Cells positive for Annexin V-FITC and negative for PI were in early apoptosis. Results were obtained from three independent experiments.

agents, however, later relapse after acquiring EGFR-TKI resistance, making it urgent to develop novel therapeutic agents to overcome acquired resistance to EGFR-TKIs $(1-3,5)$.

Gatekeeper mutations, including the T315I mutation in ABL associated with resistance to imatinib (16), the L1196M mutation in ALK associated with resistance to crizotinib (17), and the T790M mutation in EGFR associated with resistance to gefitinib and erlotinib, are common mechanisms by which tumor cells acquire resistance to molecularly targeted drugs. Although irreversible EGFR-TKIs have been developed to overcome T790M-mediated resistance to gefitinib and erlotinib, recent clinical trials have demonstrated that monotherapy with irreversible EGFR-TKIs have failed to provide benefits in patients with NSCLC refractory to gefitinib or erlotinib (18), at least in part, due to the relatively low efficacy of this class of compounds to T790M EGFR in clinically relevant concentrations.

Met amplification and/or overexpression is also associated with acquired resistance to gefitinib and erlotinib in mutated EGFR lung cancer. MET is known as the only specific receptor for hepatocyte growth factor (HGF) (19). Upon activation, MET forms a heterodimer and transduces strong signals to various pathways, including PI3K/Akt and MEK/ERK. Engelman et al (20) reported that overexpressed Met protein utilizes ErbB3 as an adaptor protein to mediate survival signals through activation of the PI3K-Akt pathway. Various targeted inhibitory strategies are being evaluated to antagonize MET/HGF signaling in human cancers, including smallmolecule kinase inhibitors, antibodies to the ligand HGF, and receptor MET itself (11). Owing to the unique intrinsic properties of MET regulating cellular 'invasive signaling', MET has been proposed as playing a role in 'oncogene addiction' 
in a small subset of human cancers as well as in 'oncogene expedience' by inducing an enhanced transformed tumor malignant 'fitness' in a much larger range of cancers, leading to promotion of tumor progression (21). In the latter case, activated MET can intercept with various other oncogenic signals, including mutant-EGFR, in maintaining and enhancing the tumor invasive-progressive phenotype, thereby also creating the opportunity for MET to be a therapeutic target even in late advanced metastatic disease $(22,23)$.

Several studies have demonstrated that the EGFR T790M mutation and MET activation have complementary roles in acquired resistance to reversible EGFR-TKI, through mediation of collaborative signaling with receptor cross-activation $(3,22)$. For example, Tang et al (21) found a direct association between MET and EGFR harboring the T790M mutation in $\mathrm{H} 1975$ cells, interaction enhanced by HGF resulting in augmented phosphorylation of Akt and ERK1/2. These observations indicate that EGFR and MET have joint downstream pro-survival signaling pathways, and activation of EGFR or MET could reciprocally trigger their downstream signaling pathways. Therefore, monotherapy with an irreversible EGFR-TKI alone not only requires high concentrations resulting in adverse effects, but also induces acquired resistance through MET activation. Accordingly, monotherapy with MET-TKI alone or MET-TKI combined with a reversible EGFR-TKI has little therapeutic effects on T790M-EGFR NSCLC cells, since pro-survival signals mediated by T790MEGFR are continuously activated in these conditions (12). Therefore, neither monotherapy is sufficient to overcome the acquired resistance. However, afatinib, a novel irreversible inhibitor of the ErbB family member EGFR, was recently shown to display preclinical efficacy in NSCLC with common EGFR-activating mutations and the T790M mutation typically associated with EGFR TKI resistance (24-26).

In the present study, we found that MET was neither genomically amplified nor mutated in the erlotinib-resistant H1975 cell line (L858R/T7980M-EGFR). Our data showed that MET had 1.1 copies in H1975 cells, in agreement with previous studies $(12,27,28)$. However, we did find that MET was activated in these cells, possessing constitutive (ligandindependent) receptor activation. The double activation of MET and EGFR not only conferred resistance to erlotinib but also resulted in markedly enhanced catalytic kinases, demonstrated in cell cytotoxicity assays and western blot analysis of EGFR, MET and their downstream signaling pathways in agreement with previous reports (19). When treating H1975 cells with different inhibitors, BIBW2992 and ARQ 197 alone and the erlotinib/ARQ 197 combination showed only little or moderate effects on cell growth inhibition, apoptosis induction and cell cycle arrest. BIBW2992 and ARQ 197 decreased EGFR and MET phosphorylation, respectively. However, BIBW2992 or ARQ 197 alone had no impact on AKT and ERK1/2 phosphorylation. In contrast, the combination of BIBW2992 and ARQ 197 showed pronounced effects on cell growth inhibition, apoptosis induction and cell cycle arrest, and markedly decreased phosphorylation of AKT and ERK1/2. The therapeutic effects of BIBW2992 combined with ARQ 197 were more evident than that of the erlotinib/ARQ 197 combination as shown above. This might result from stronger cytotoxic effects of BIBW2992 compared with reversible EGFR-TKIs in T790M-EGFR NSCLC cells. Finally, dual MET and EGFR siRNA knockdown experiments in H1975 cells provided further validation of the therapeutic value of an irreversible EGFR-TKI combined with MET-TKI. Dual inhibition appears to be superior to single target inhibition. Our present report demonstrated the efficacy of dual receptor tyrosine kinasetargeted inhibition against EGFR (BIBW2992) and MET (ARQ 197) as a strategy to achieve optimized inhibition of cell viability, cell cycle progression and cellular signaling in T790M-EGFR-mediated erlotinib resistance in H1975 cells.

Overall, our findings suggest that the combination of MET-TKI and irreversible EGFR-TKI may be effective in controlling H1975 cells with acquired resistance to erlotinib. Downregulation of the PI3K/AKT and MEK/ERK signaling pathways were related to the cytotoxic effects of this combinational therapeutic approach. Further studies should focus on the exact mechanisms of apoptosis induction and cross-talk between EGFR and MET downstream pro-survival signaling pathways.

\section{References}

1. Vincent MD, Kuruvilla MS, Leighl NB and Kamel-Reid S: Biomarkers that currently affect clinical practice: EGFR, ALK, MET, KRAS. Curr Oncol 19: S33-S44, 2012.

2. Capelletto E and Novello S: Emerging new agents for the management of patients with non-small cell lung cancer. Drugs 72 (Suppl 1): 37-52, 2012.

3. Suda K, Mizuuchi H, Maehara Y and Mitsudomi T: Acquired resistance mechanisms to tyrosine kinase inhibitors in lung cancer with activating epidermal growth factor receptor mutation - diversity, ductility, and destiny. Cancer Metastasis Rev 31: 807-814, 2012.

4. Liu Y, Shi QF, Ye YC, Tashiro S, Onodera S and Ikejima T: Activated $\mathrm{O}_{2}^{-}$and $\mathrm{H}_{2} \mathrm{O}_{2}$ mediated cell survival in SU11274treated non-small-cell lung cancer A549 cells via c-Met-PI3K-Akt and c-Met-Grb2/SOS-Ras-p38 pathways. J Pharmacol Sci 119: 150-159, 2012.

5. Ayoola A, Barochia A, Belani K and Belani CP: Primary and acquired resistance to epidermal growth factor receptor tyrosine kinase inhibitors in non-small cell lung cancer: an update. Cancer Invest 30: 433-446, 2012.

6. Xu L, Kikuchi E, Xu C, et al: Combined EGFR/MET or EGFR/ HSP90 inhibition is effective in the treatment of lung cancers codriven by mutant EGFR containing T790M and MET. Cancer Res 72: 3302-3311, 2012.

7. Li D, Ambrogio L, Shimamura T, et al: BIBW2992, an irreversible EGFR/HER2 inhibitor highly effective in preclinical lung cancer models. Oncogene 27: 4702-4711, 2008.

8. Takezawa K, Okamoto I, Tanizaki J, et al: Enhanced anticancer effect of the combination of BIBW2992 and thymidylate synthase-targeted agents in non-small cell lung cancer with the T790M mutation of epidermal growth factor receptor. Mol Cancer Ther 9: 1647-1656, 2010.

9. Dulak AM, Gubish CT, Stabile LP, Henry C and Siegfried JM: HGF-independent potentiation of EGFR action by c-Met. Oncogene 30: 3625-3635, 2011.

10. Nakachi I, Naoki K, Soejima K, et al: The combination of multiple receptor tyrosine kinase inhibitor and mammalian target of rapamycin inhibitor overcomes erlotinib resistance in lung cancer cell lines through c-Met inhibition. Mol Cancer Res 8: 1142-1151, 2010 .

11. Liu X, Newton RC and Scherle PA: Development of c-MET pathway inhibitors. Expert Opin Investig Drugs 20: 1225-1241, 2011.

12. Nakagawa T, Takeuchi S, Yamada T, et al: Combined therapy with mutant-selective EGFR inhibitor and Met kinase inhibitor for overcoming erlotinib resistance in EGFR-mutant lung cancer. Mol Cancer Ther 11: 2149-2157, 2012.

13. Comoglio PM, Giordano S and Trusolino L: Drug development of MET inhibitors: targeting oncogene addiction and expedience. Nat Rev Drug Discov 7: 504-516, 2008. 
14. Brambilla E and Gazdar A: Pathogenesis of lung cancer signalling pathways: roadmap for therapies. Eur Respir J 33: 1485-1497, 2009.

15. Suda K, Tomizawa K, Osada $\mathrm{H}$, et al: Conversion from the 'oncogene addiction' to 'drug addiction' by intensive inhibition of the EGFR and MET in lung cancer with activating EGFR mutation. Lung Cancer 76: 292-299, 2012.

16. Branford S, Rudzki Z, Walsh S, et al: High frequency of point mutations clustered within the adenosine triphosphate-binding region of $\mathrm{BCR} / \mathrm{ABL}$ in patients with chronic myeloid leukemia or Ph-positive acute lymphoblastic leukemia who develop imatinib (STI571) resistance. Blood 99: 3472-3475, 2002.

17. Choi YL, Soda M, Yamashita Y, et al: EML4-ALK mutations in lung cancer that confer resistance to ALK inhibitors. N Engl J Med 363: 1734-1739, 2010.

18. Wong KK, Fracasso PM, Bukowski RM, et al: A phase I study with neratinib (HKI-272), an irreversible pan ErbB receptor tyrosine kinase inhibitor, in patients with solid tumors. Clin Cancer Res 15: 2552-2558, 2009.

19. Guo A, Villen J, Kornhauser J, et al: Signaling networks assembled by oncogenic EGFR and c-Met. Proc Natl Acad Sci USA 105: 692-697, 2008.

20. Engelman JA, Zejnullahu K, Mitsudomi T, et al: MET amplification leads to gefitinib resistance in lung cancer by activating ERBB3 signaling. Science 316: 1039-1043, 2007.

21. Tang Z, Du R, Jiang S, et al: Dual MET-EGFR combinatorial inhibition against T790M-EGFR-mediated erlotinib-resistant lung cancer. Br J Cancer 99: 911-922, 2008.
22. Suda K, Murakami I, Katayama T, et al: Reciprocal and complementary role of $M E T$ amplification and EGFR T790M mutation in acquired resistance to kinase inhibitors in lung cancer. Clin Cancer Res 16: 5489-5498, 2010.

23. Bonanno L, Jirillo A and Favaretto A: Mechanisms of acquired resistance to epidermal growth factor receptor tyrosine kinase inhibitors and new therapeutic perspectives in non small cell lung cancer. Curr Drug Targets 12: 922-933, 2011.

24. Bowles DW, Weickhardt A and Jimeno A: Afatinib for the treatment of patients with EGFR-positive non-small cell lung cancer. Drugs Today 49: 523-535, 2013.

25. Nelson V,Ziehr J, Agulnik M and Johnson M: Afatinib: emerging next-generation tyrosine kinase inhibitor for NSCLC. Onco Targets Ther 6: 135-143, 2013.

26. Chen X, Zhu Q, Zhu L, et al: Clinical perspective of afatinib in non-small cell lung cancer. Lung Cancer 81: 155-161, 2013.

27. Wang W, Li Q, Takeuchi S, et al: Met kinase inhibitor E7050 reverses three different mechanisms of hepatocyte growth factorinduced tyrosine kinase inhibitor resistance in EGFR mutant lung cancer. Clin Cancer Res 18: 1663-1671, 2012.

28. Koizumi H, Yamada T, Takeuchi S, et al: Hsp90 inhibition overcomes HGF-triggering resistance to EGFR-TKIs in EGFRmutant lung cancer by decreasing client protein expression and angiogenesis. J Thorac Oncol 7: 1078-1085, 2012. 